\title{
Field Evaporation Characteristics in Hafnium Carbides
}

\author{
F. Vogel ${ }^{1,2,3}$, S. Ngai ${ }^{1,4}$, C. Smith ${ }^{1} \&$ G.B. Thompson ${ }^{1}$ \\ ${ }^{1}$ University of Alabama, Department of Metallurgical and Materials Engineering, Tuscaloosa, AL, \\ 34587 USA \\ 2 Technische Universität Berlin, Department of Materials Science and Technology, Hardenberg-Str. 36, \\ 10623 Berlin, Germany \\ 3 Helmholtz-Zentrum Berlin, Institute of Applied Materials, Hahn-Meitner-Platz 1, 14109 Berlin, \\ Germany \\ 4 South China University of Technology, School of Mechanical and Automobile Engineering, \\ Guangzhou 510640, P.R. China
}

The use of pulsed lasers has enabled the field evaporation of lower conductivity materials, including semiconductors and ceramics, in the atom probe [1-2]. The variability of laser power, tip shape, and composition can have a profound effect on this field evaporation. In this presentation, these variables have been systematically adjusted to ascertain how the field evaporation evolves in hafnium carbide. The field evaporation experiments were carried out in a Cameca Local Electrode Atom Probe (LEAP) 5000 XS.

Hafnium carbide (HfC) is a rock salt crystal structure (B1) and has a melting temperature near $4000^{\circ} \mathrm{C}$, which is one of the highest known melting temperatures for a material. The loss of carbon in HfC is predicated to form a series of sub-stoichiometric vacancy ordered phases [3]; however, in practice, these phases rarely form because of the difficulty to vacancy order in this ceramic and the B1 phase is retained. By removing carbon from the octahedral interstitial sites of the FCC lattice, the ceramic becomes more metallic in character while retaining this B1 phase. This provides a distinctive opportunity to investigate how field evaporation changes in a ceramic whose composition varies but the crystallography is retained.

Figure 1 is a detector event histogram (DEH) that describes the evolution of evaporation strikes for various laser pulse energies from 400 to $1200 \mathrm{pJ}$. At the lower energies, a <100> pole is visible. As the laser pulse energy increased, particularly at $1200 \mathrm{pJ}$, the DEH became more uniform, though remnants of the influence of the pole are still apparent. This increase in laser power resulted in a linear rise of single hits from $58 \%$ to $96 \%$ for the same specimen tip along with the field evaporation of more complex species in the mass-to-charge spectrum, Figure 2.

Figure 2 is a representative region between 160-205 Da where the onset of $\mathrm{HfC}^{2+}$ and $\mathrm{HfCN}^{+}$peaks became apparent with increasing laser pulse energy. In addition, the increased laser pulse energy enabled the resolution of the $\mathrm{Hf}^{+}$peaks between 175 to 180 Da from the prior broad evaporation 'hump.' Though the increased laser pulse energy is beneficial in many ways, measurements of the mass resolving power (MRP) were optimal at $1000 \mathrm{pJ}$, with a MRP of $~ 850$ for ${ }^{180} \mathrm{Hf}^{2+}$. If $1200 \mathrm{pJ}$ was used, the MRP for this peak decreased to $\sim 600$. Finally, the Hf:C composition varied with the laser pulse energy settings, with increasing power resulting in Hf-rich compositions, which has been noted to occur in other metal-carbides [2]. 


\section{References:}

[1] T.F. Kelly et al. Annu. Rev. Mater. Res. 37 (2007) 681-727

[2] M. Thuvander et al. Ultramicroscopy 111 (2011) 604-608

[3] X.X. Yu et al., Computational Materials Science 112 (2016) 318-326

[4] The authors gratefully recognize support from the Air Force Office of Scientific Research grant FA9550-15-1-0095, Dr. Ali Sayir program manager, as well as the University of Alabama's Central Analytical Facility.
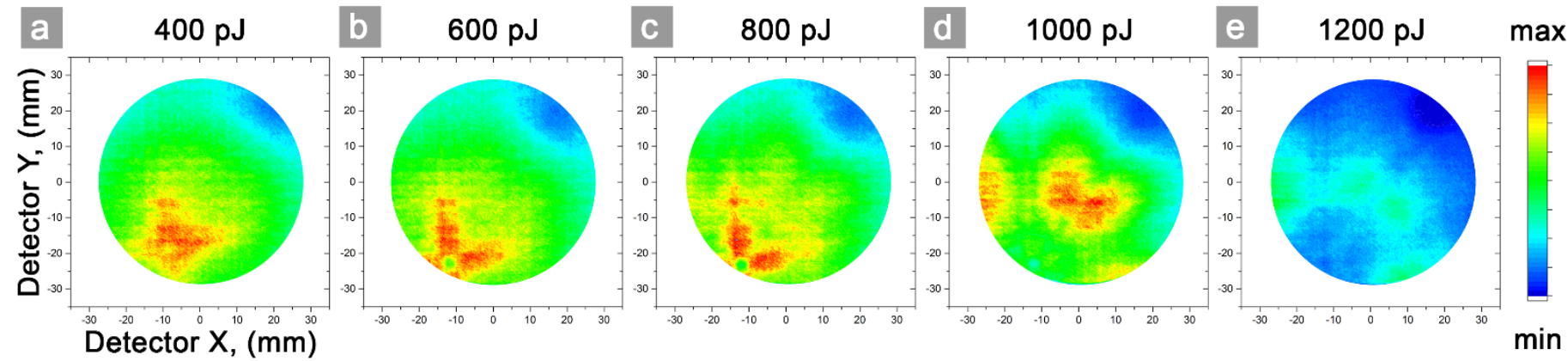

Figure 1: Evolution of detector hit histogram for various laser pulse energies, (a) 400 pJ, (b) 600 pJ, (c) 800 pJ, (d) 1000 pJ and (e) 1200 pJ.

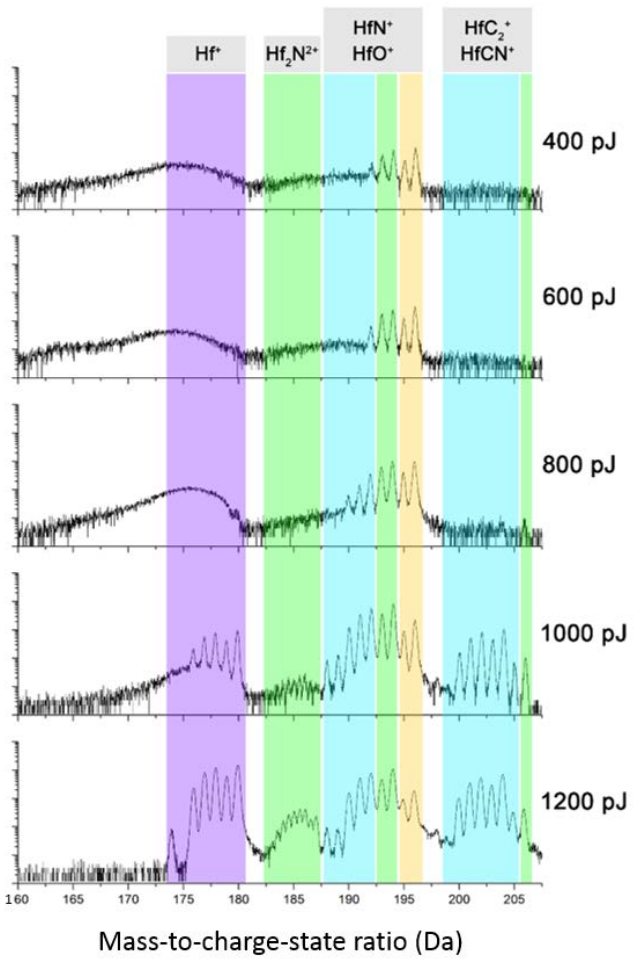

Figure 2: A section of a mass spectrum analyses of APT datasets acquired from $\mathrm{HfC}_{0.8}$ at laser pulse energies of 400-1200 pJ but otherwise constant parameters. The ranges corresponding to $\mathrm{Hf}$ and $\mathrm{Hf}_{2}$ (purple), $\mathrm{HfN}$ and $\mathrm{HfO}$ (green) and $\mathrm{HfC}_{\mathrm{x}}$ (blue) for singly and douply charged states are indicated 